\title{
The evolution of uniform random planar graphs
}

\author{
Chris Dowden \\ LIX, École Polytechnique, 91128 Palaiseau Cedex, France \\ dowden@lix.polytechnique.fr \\ Submitted: Jun 2, 2009; Accepted: Dec 18, 2009; Published: Jan 5, 2010 \\ Mathematics Subject Classification: 05C10, 05C80
}

\begin{abstract}
Let $P_{n, m}$ denote the graph taken uniformly at random from the set of all planar graphs on $\{1,2, \ldots, n\}$ with exactly $m(n)$ edges. We use counting arguments to investigate the probability that $P_{n, m}$ will contain given components and subgraphs, finding that there is different asymptotic behaviour depending on the ratio $\frac{m}{n}$.
\end{abstract}

\section{Introduction}

Random planar graphs have recently been the subject of much activity, and many properties of the standard random planar graph $P_{n}$ (taken uniformly at random from the set of all planar graphs on $\{1,2, \ldots, n\}$ ) are now known. For example, in [7] it was shown that $P_{n}$ will asymptotically almost surely (a.a.s., that is, with probability tending to 1 as $n$ tends to infinity) contain at least linearly many copies of any given planar graph. By combining the counting methods of [7] with some rather precise results of [5], obtained using generating functions, the exact limiting probability for the event that $P_{n}$ will contain any given component is also known.

More recently, attention has turned to the graph $P_{n, m}$ taken uniformly at random from the set $\mathcal{P}(n, m)$ of all planar graphs on $\{1,2, \ldots, n\}$ with exactly $m(n)$ edges. It is well known that we must have $m<3 n$ for planarity to be possible and also that $P_{n, m}$ behaves in exactly the same way as the general random graph $G_{n, m}$ if $\frac{m}{n}<\frac{n}{2}-\omega\left(n^{2 / 3}\right)$ (see, for example, [6]), so the interest lies with the region $\frac{1}{2} \leqslant \lim \inf \frac{m}{n} \leqslant \lim \sup \frac{m}{n} \leqslant 3$.

In [4], the case when $m=\lfloor q n\rfloor$ for fixed $q$ was investigated using counting arguments and it was shown that, for all $q \in(1,3), P_{n,\lfloor q n\rfloor}$ will a.a.s. contain at least linearly many copies of any given planar graph, as with $P_{n}$. It was also shown that the probability that $P_{n,\lfloor q n\rfloor}$ will contain an isolated vertex is bounded away from 0 as $n \rightarrow \infty$ (for all $q<3$ ) and hence that the probability that $P_{n,\lfloor q n\rfloor}$ will be connected is bounded away from 1 . For $q \in(1,3)$, the precise limit for $\mathbf{P}\left[P_{n,\lfloor q n\rfloor}\right.$ will be connected] may be obtained from a later result in [5], which uses a generating function approach. 
As already mentioned, the exact limiting probability for the event that $P_{n}$ will contain any given component was determined by combining results of [5] and [7]. Unfortunately, although the basic method for this does generalise to $P_{n,\lfloor q n\rfloor}$, some difficulties arise in the details of the equations, and it may well be the case that the probability does not usually converge to a limit here.

In this paper, we use counting arguments to extend the current knowledge of $P_{n, m}$. We investigate the probability that $P_{n, m}$ will contain given components and the probability that $P_{n, m}$ will contain given subgraphs, both for general $m(n)$, and show that there is different behaviour depending on which 'region' the ratio $\frac{m(n)}{n}$ falls into. Hence, this change as $\frac{m}{n}$ varies can be thought of as the 'evolution' of uniform random planar graphs.

We start in Section 2 by collecting up various lemmas on $P_{n, m}$ that will prove useful to us. In Section 3, we then obtain lower bounds for $\mathbf{P}:=\mathbf{P}\left[P_{n, m}\right.$ will have a component isomorphic to $H$ ] (where by 'lower bound' we mean a result such as $\lim \inf \mathbf{P}>0$ or $\mathbf{P} \rightarrow 1$ ), and in Section 4 we produce exactly complementary upper bounds. Finally, in Section 5, we look at the probability that $P_{n, m}$ will have a copy of $H$ (i.e. any subgraph isomorphic to $H$ ).

A summary of our results is given in Tables 1 and 2. These tables both have three columns, corresponding to the sign of $e(H)-|H|$ (the excess of edges over vertices), and also different rows, corresponding to the size of $\frac{m(n)}{n}$. We use $\underline{\text { lim }}$ to denote lim inf and $\varlimsup$ lim to denote limsup, and 'T8' (for example) refers to Theorem 8.

\begin{tabular}{|c|c|c|c|}
\hline & $e(H)<|H|$ & $e(H)=|H|$ & $e(H)>|H|$ \\
\hline $0<\underline{\lim } \frac{m}{n}$ & $\mathbf{P} \rightarrow 1(\mathrm{Thm} 10)$ & $\underline{\lim } \mathbf{P}>0(\mathrm{~T} 9)$ & $\mathbf{P} \rightarrow 0(\mathrm{Thm} 12)$ \\
$\& \frac{m}{n} \leqslant 1+o(1)$ & & $\mathbf{\operatorname { l i m }} \mathbf{P}<1(\mathrm{~T} 13)$ & \\
\hline $1<\underline{\lim } \frac{m}{n}$ & $\underline{\lim } \mathbf{P}>0(\mathrm{~T} 8)$ & $\underline{\lim } \mathbf{P}>0(\mathrm{~T} 8)$ & $\underline{\lim } \mathbf{P}>0(\mathrm{~T} 8)$ \\
$\& \lim \frac{m}{n}<3$ & $\lim _{\mathbf{P}}<1(\mathrm{~T} 16)$ & $\mathbf{\operatorname { l i m }}<1(\mathrm{~T} 16)$ & $\mathbf{\operatorname { l i m }}<1(\mathrm{~T} 16)$ \\
\hline$\frac{m}{n} \rightarrow 3$ & $\mathbf{P} \rightarrow 0(\mathrm{Thm} 14)$ & $\mathbf{P} \rightarrow 0(\mathrm{Thm} 14)$ & $\mathbf{P} \rightarrow 0(\mathrm{Thm} 14)$ \\
\hline
\end{tabular}

Table 1: A description of $\mathbf{P}:=\mathbf{P}\left[P_{n, m}\right.$ will have a component isomorphic to $\left.H\right]$.

\begin{tabular}{|c|c|c|c|}
\hline & $\overline{e(H)<|H|}$ & $\overline{e(H)}=|H|$ & $\overline{e(H)>|H|}$ \\
\hline $\begin{array}{c}0<\underline{\lim } \frac{m}{n} \\
\& \varlimsup \frac{m}{n}<1\end{array}$ & $\mathbf{P}^{\prime} \rightarrow 1($ Thm 10 & $\begin{array}{l}\underline{\underline{\lim }} \mathbf{P}^{\prime}>0(\mathrm{~T} 9) \\
\lim \mathbf{P}^{\prime}<1(\mathrm{~T} 18)\end{array}$ & $\mathbf{P}^{\prime} \rightarrow 0($ Thm 22) \\
\hline$\frac{m}{n}-$ & $\mathbf{P}^{\prime} \rightarrow 1$ & $\mathbf{P}^{\prime} \rightarrow 1($ Thm 21) & \\
\hline$\underline{\underline{\lim }} \frac{m}{n}>1$ & $\mathbf{P}^{\prime} \rightarrow 1($ Thm 17$)$ & $\mathbf{P}^{\prime} \rightarrow 1($ Thm 17$)$ & $\mathbf{P}^{\prime} \rightarrow 1$ \\
\hline
\end{tabular}

Table 2: A description of $\mathbf{P}^{\prime}:=\mathbf{P}\left[P_{n, m}\right.$ will have a copy of $\left.H\right]$. 


\section{Appearances, Pendant Edges \& Addable Edges}

In this section, we shall lay the groundwork for the rest of the paper by noting some useful properties of $P_{n, m}$. We will see results on the number of 'appearances' (special subgraphs) in $P_{n, m}$, the number of 'pendant' edges (i.e. edges incident to a vertex of degree 1), and the number of 'addable' edges (i.e. edges that can be added to $P_{n, m}$ without violating planarity). All of these will be important ingredients in the counting arguments of later sections.

We start with the definition of an appearance:

Definition 1 Let $H$ be a graph on the vertex set $\{1,2, \ldots,|H|\}$, and let $G$ be a graph on the vertex set $\{1,2, \ldots, n\}$, where $n>|H|$. Let $W \subset V(G)$ with $|W|=|H|$, and let the 'root' $r_{W}$ denote the least element in $W$. We say that $H$ appears at $W$ in $G$ if (a) the increasing bijection from $1,2, \ldots,|H|$ to $W$ gives an isomorphism between $H$ and the induced subgraph $G[W]$ of $G$; and (b) there is exactly one edge in $G$ between $W$ and the rest of $G$, and this edge is incident with the root $r_{W}$ (see Figure 1 ). We let $f_{H}(G)$ denote the number of appearances of $H$ in $G$, that is the number of sets $W \subset V(G)$ such that $H$ appears at $W$ in $G$.

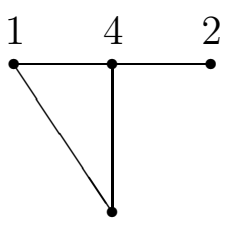

3

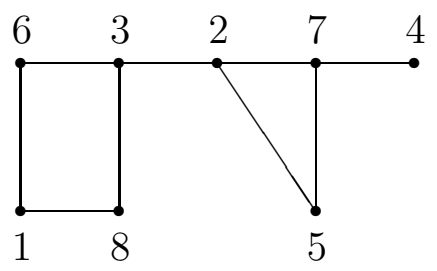

Figure 1: A graph $H$ and an appearance of $H$.

The following result on appearances was given in [4]:

Proposition 2 ([4], Theorem 3.1) Let $H$ be a (fixed) connected planar graph on the vertices $\{1,2, \ldots,|H|\}$ and let $q \in(1,3)$ be a constant. Then there exists a constant $\alpha(H, q)>0$ such that

$$
\mathbf{P}\left[f_{H}\left(P_{n,\lfloor q n\rfloor}\right) \leqslant \alpha n\right]=e^{-\Omega(n)} .
$$

It is, in fact, fairly easy to deduce from the proof of Proposition 2 given in [4] that the result holds uniformly in $q$ (see [1] for details). Hence, we may actually obtain the following stronger version:

Lemma 3 ([1], Lemma 13) Let $H$ be a (fixed) connected planar graph on the vertices $\{1,2, \ldots,|H|\}$, let $b>1$ and $B<3$ be constants, and let $m(n) \in[b n, B n]$ for all $n$. Then there exists a constant $\alpha=\alpha(H, b, B)>0$ such that

$$
\mathbf{P}\left[f_{H}\left(P_{n, m}\right) \leqslant \alpha n\right]=e^{-\Omega(n)} .
$$


An important consequence of Lemma 3 is that $P_{n, m}$ will a.a.s. contain a copy of any given planar graph if $1<\liminf \frac{m}{n} \leqslant \limsup \frac{m}{n}<3$ (as noted in the introduction). However, it is the precise uncomplicated structure of appearances themselves that will be particularly useful to us during this paper.

Next, let us note that it follows from Lemma 3 (with $H$ as an isolated vertex) that $P_{n, m}$ will a.a.s. have linearly many pendant edges if $1<\liminf \frac{m}{n} \leqslant \lim \sup \frac{m}{n}<3$. It is fairly intuitive that we ought to be able to drop the lower bound to $\lim$ inf $\frac{m}{n}>0$ for this particular case, and this is indeed shown in [1]. Hence, we obtain:

Lemma 4 ([1], Theorem 16) Let $b>0$ and $B<3$ be constants and let $m(n) \in[b n, B n]$ for all $n$. Then there exists a constant $\alpha=\alpha(b, B)>0$ such that

$$
\mathbf{P}\left[P_{n, m} \text { will have less than } \alpha \text { pendant edges }\right]=e^{-\Omega(n)} \text {. }
$$

We now move on to the final topic of this section, that of 'addable' edges:

Definition 5 Given a planar graph $G$, we call a non-edge e addable in $G$ if the graph $G+e$ obtained by adding $e$ as an edge is still planar. We let $\operatorname{add}(G)$ denote the set of addable non-edges of $G$ (note that the graph obtained by adding two edges in $\operatorname{add}(G)$ may well not be planar) and we let $\operatorname{add}(n, m)$ denote the minimum value of $|\operatorname{add}(G)|$ over all graphs $G \in \mathcal{P}(n, m)$.

In future sections, we shall often wish to choose an edge to insert into a graph without violating planarity, and we will want to know how many choices we have. A very helpful result is given implicitly in Theorem 1.2 of [2]:

Lemma 6 ([2], Theorem 1.2) Let $m(n) \leqslant(1+o(1)) n$. Then

$$
\operatorname{add}(n, m)=\omega(n),
$$

i.e. $\frac{\operatorname{add}(n, m)}{n} \rightarrow \infty$ as $n \rightarrow \infty$.

We should also note that a useful higher estimate for the case $\lim \sup \frac{m}{n}<1$ can be obtained very simply:

Lemma 7 Let $A<1$ be a constant and let $m(n) \leqslant A n$ for all $n$. Then

$$
\operatorname{add}(n, m) \geqslant(1+o(1))\left(\frac{(1-A)^{2}}{2}\right) n^{2} .
$$

Proof Any graph in $\mathcal{P}(n, m)$ must have at least $n-m=(1-A) n$ components, and it is known that inserting an edge between any two vertices in different components will not violate planarity. Hence, $\operatorname{add}(n, m) \geqslant\left(\begin{array}{c}(1-A) n \\ 2\end{array}\right)$. 


\section{Components I: Lower Bounds}

We now come to the first main section of this paper, where we shall start to use the results of Section 2 to investigate $\mathbf{P}:=\mathbf{P}\left[P_{n, m}\right.$ will have a component isomorphic to $H]$. We shall first see (in Theorem 8) that $\lim \inf \mathbf{P}>0$ for all connected planar $H$ if $1<\liminf \frac{m}{n} \leqslant \limsup \frac{m}{n}<3$, then (in Theorem 9) that the lower bound on $\frac{m}{n}$ can be reduced to $\liminf \frac{m}{n}>0$ if $e(H) \leqslant|H|$, and thirdly (in Theorem 10) that $\mathbf{P} \rightarrow 1$ if $0<\liminf \frac{m}{n} \leqslant \limsup \frac{m}{n} \leqslant 1$ and $H$ is a tree. Finally, we will show (in Theorem 11) that $P_{n, m}$ will a.a.s. have linearly many components isomorphic to any given tree if $0<\liminf \frac{m}{n} \leqslant \lim \sup \frac{m}{n}<1$.

We start with our aforementioned result for general connected planar $H$ :

Theorem 8 Let $H$ be a (fixed) connected planar graph, let $b>1$ and $B<3$ be constants, and let $m(n) \in[b n, B n]$ for all $n$. Then there exist constants $\epsilon(H, b, B)>0$ and $N(H, b, B)$ such that

$$
\mathbf{P}\left[P_{n, m} \text { will have a component isomorphic to } H\right] \geqslant \epsilon \text { for all } n \geqslant N \text {. }
$$

Sketch of Proof We shall suppose that the result is false. Thus, there exist arbitrarily large values of $n$ for which a typical graph in $\mathcal{P}(n, m)$ will have no components isomorphic to $H$, but will have many appearances of $K_{4}$ (by Lemma 3). From each such graph, we shall construct graphs in $\mathcal{P}(n, m)$ that do have a component isomorphic to $H$.

We start by deleting edges from some of our appearances of $K_{4}$ to create isolated vertices, on which we then build a component isomorphic to $H$. By inserting extra edges in appropriate places elsewhere, we hence obtain graphs that are also in $\mathcal{P}(n, m)$. The fact that the original graphs contained no components isomorphic to $H$ can then be used to show that there isn't too much double-counting, and so we find that we have actually constructed a decent number of distinct graphs in $\mathcal{P}(n, m)$ that have components isomorphic to $H$, which is what we wanted to prove.

Full Proof Let $\epsilon \in(0,1)$. Since $\frac{m}{n} \in[b, B]$ for all $n$, by Lemma 3 there exist constants $\alpha=\alpha(b, B)>0$ and $N(b, B)$ such that, for all $n \geqslant N, \mathbf{P}\left[P_{n, m}\right.$ will have at least $\alpha n$ appearances of $\left.K_{4}\right] \geqslant\left(1-\frac{\epsilon}{2}\right)$. Note that any appearances of $K_{4}$ must be vertex-disjoint, by 2-edge-connectedness.

Consider an $n \geqslant N$ and suppose that $\mathbf{P}\left[P_{n, m}\right.$ will have a component isomorphic to $H]<1-\epsilon$ (if not, then we are certainly done). Let $\mathcal{G}_{n}$ denote the set of graphs in $\mathcal{P}(n, m)$ with (i) no components isomorphic to $H$ and (ii) at least $\alpha n$ vertex-disjoint appearances of $K_{4}$. Then, under our assumption, we have $\left|\mathcal{G}_{n}\right| \geqslant \frac{\epsilon}{2}|\mathcal{P}(n, m)|$. We shall use $\mathcal{G}_{n}$ to construct graphs in $\mathcal{P}(n, m)$ that do have a component isomorphic to $H$.

Consider a graph $G \in \mathcal{G}_{n}$. We may assume that $n$ is large enough that $\alpha n \geqslant|H|$. Thus, we may choose $|H|$ of the (vertex-disjoint) appearances of $K_{4}$ in $G$ (at least $\left(\begin{array}{c}\lceil\alpha n\rceil \\ |H|\end{array}\right)$ choices), and for each of these chosen appearances we may choose a 'special' vertex in the $K_{4}$ that is not the root $\left(3^{|H|}\right.$ choices $)$. Let us then delete all $3|H|$ edges that are incident to the 
'special' vertices and insert edges between these $|H|$ newly isolated vertices in such a way that they now form a component isomorphic to $H$ (see Figure 2).

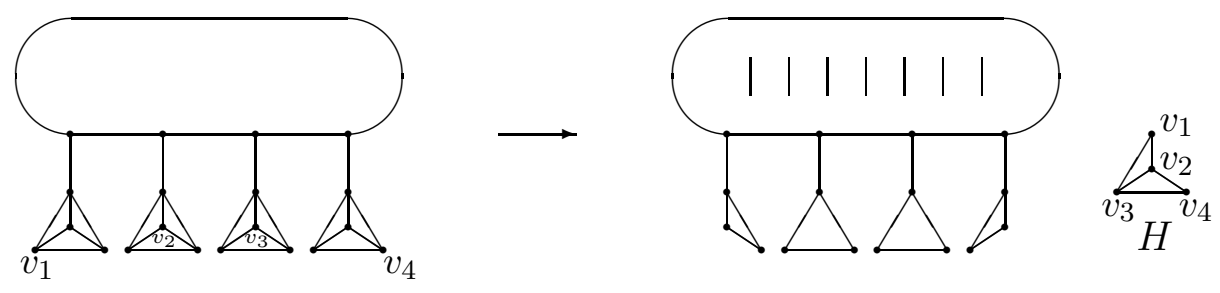

Figure 2: Constructing a component isomorphic to $H$.

To maintain the correct number of edges, we should insert $3|H|-e(H)$ extra ones somewhere into the graph, making sure that we maintain planarity. We will do this in such a way that we do not interfere with our new component or with the chosen appearances of $K_{4}$ (which are now appearances of $K_{3}$ ). Thus, the part of the graph where we wish to insert edges contains $n-4|H|$ vertices and $m-7|H|$ edges. We know that there exists a triangulation on these vertices containing these edges, and clearly inserting an edge from this triangulation would not violate planarity. Thus, we have at least $\left(\begin{array}{c}3(n-4|H|)-6-(m-7|H|) \\ 3|H|-e(H)\end{array}\right)$ choices for where to add the edges.

Therefore, in total we find that we have at least $\left|\mathcal{G}_{n}\right|\left(\begin{array}{c}\lceil\alpha n\rceil \\ |H|\end{array}\right) 3^{|H|}\left(\begin{array}{c}3(n-4|H|)-6-(m-7|H|) \\ 3|H|-e(H)\end{array}\right)=$ $\left|\mathcal{G}_{n}\right| \Theta\left(n^{4|H|-e(H)}\right)$ ways to build (not necessarily distinct) graphs in $\mathcal{P}(n, m)$ that have a component isomorphic to $H$.

We will now consider the amount of double-counting:

Each of our constructed graphs will contain at most $4|H|-e(H)+1$ components isomorphic to $H$ (since there were none originally; we have deliberately built one; and we may have created at most one extra one each time we cut a 'special' vertex away from its $K_{4}$ or added an edge in the rest of the graph). Hence, we have at most $4|H|-e(H)+1$ possibilities for which were our $|H|$ 'special' vertices. Since appearances of $K_{3}$ must be vertex-disjoint, by 2-edge-connectedness, we have at most $\frac{n}{3}$ of them and hence at most $\left(\frac{n}{3}\right)^{|H|}$ possibilities for where the 'special' vertices were originally. There are then at most $\left(\begin{array}{c}m-e(H)-4|H| \\ 3|H|-e(H)\end{array}\right)$ possibilities for which edges were added in the rest of the graph (i.e. away from the constructed component isomorphic to $H$ and these appearances of $K_{3}$ ). Thus, the amount of double-counting is at most $(4|H|-e(H)+1)\left(\frac{n}{3}\right)^{|H|}\left(\begin{array}{c}m-e(H)-4|H| \\ 3|H|-e(H)\end{array}\right)=\Theta\left(n^{4|H|-e(H)}\right)$, recalling that $m=\Theta(n)$.

Hence, we find that the number of distinct graphs that we have constructed is at least $\frac{\left|\mathcal{G}_{n}\right| \Theta\left(n^{4|H|-e(H)}\right)}{\Theta\left(n^{4|H|-e(H)}\right)}=\left|\mathcal{G}_{n}\right| \Theta(1)$. Thus, recalling that $\left|\mathcal{G}_{n}\right| \geqslant \frac{\epsilon}{2}|\mathcal{P}(n, m)|$, we are done.

Note that in the previous proof, we could have constructed a component isomorphic to $H$ directly from an appearance of $H$. We chose to instead build the component from isolated vertices cut from appearances of $K_{4}$, as this technique generalises more easily to 
our next proof, as we shall now explain.

Recall that when we cut the isolated vertices from the appearances of $K_{4}$, this involved deleting three edges for each isolated vertex that we created, which crucially meant that we had enough edges to play with when we wanted to turn these isolated vertices into a component isomorphic to $H$. Notice, though, that the proof was only made possible by the fact that we had lots of appearances of $K_{4}$ to choose from, which was why we needed to restrict $\frac{m}{n}$ to the region $[b, B]$, where $b>1$ and $B<3$.

However, if $e(H) \leqslant|H|$ then we would have enough edges to play with even if we only deleted one edge for each isolated vertex that we created. Thus, we may replace the role of the appearances of $K_{4}$ by pendant edges, which we know are plentiful even for small values of $\frac{m}{n}$, by Lemma 4 . Hence, we may obtain:

Theorem 9 Let $H$ be a (fixed) connected planar graph with $e(H) \leqslant|H|$, let $c>0$ and $B<3$ be constants, and let $m(n) \in[c n, B n]$ for all $n$. Then there exist constants $\epsilon(H, c, B)>0$ and $N(H, c, B)$ such that

$$
\mathbf{P}\left[P_{n, m} \text { will have a component isomorphic to } H\right] \geqslant \epsilon \text { for all } n \geqslant N \text {. }
$$

Proof Suppose the result is false. Then, similarly to with the proof of Theorem 8, we have a set $\mathcal{G}_{n}$ of at least $\frac{\epsilon}{2}|\mathcal{P}(n, m)|$ graphs with (i) no components isomorphic to $H$ and (ii) at least $\alpha$ pendant edges (using Lemma 4).

Given a graph $G \in \mathcal{G}_{n}$, we may delete $|H|$ of the pendant edges and use the resulting isolated vertices to construct a component isomorphic to $H$ (see Figure 3). If $H$ is a tree, then we should also add one edge in a suitable place somewhere in the rest of the graph.
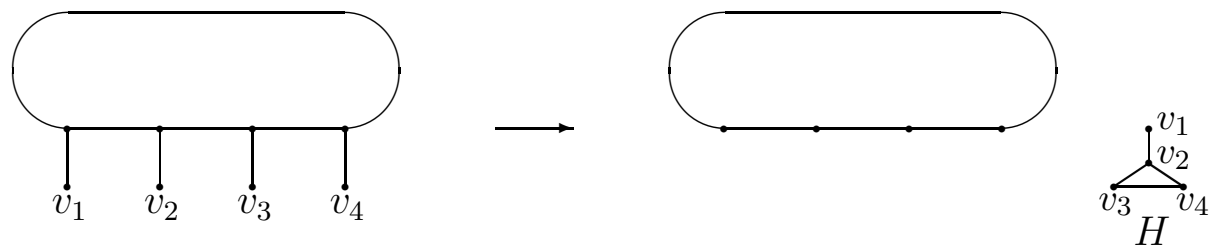

Figure 3: Constructing a component isomorphic to $H$.

By similar counting arguments to those used in the proof of Theorem 8, we achieve our result.

By exactly the same proof as for Theorem 9, using the additional ingredient that $\operatorname{add}(n, m)=\omega(n)$ if $\frac{m}{n} \leqslant 1+o(1)$ (from Lemma 6), we also obtain our third result of this section:

Theorem 10 Let $H$ be a (fixed) tree, let $c>0$ be a constant, and let $m(n) \in[$ cn, $(1+$ $o(1)) n]$ as $n \rightarrow \infty$. Then

$$
\mathbf{P}\left[P_{n, m} \text { will have a component isomorphic to } H\right] \rightarrow 1 \text { as } n \rightarrow \infty \text {. }
$$


Proof As before, we suppose that $\mathbf{P}\left[P_{n, m}\right.$ will have a component isomorphic to $\left.H\right]<1-\epsilon$ for an arbitrary $\epsilon \in(0,1)$, and that we hence have a set $\mathcal{G}_{n}$ of at least $\frac{\epsilon}{2}|\mathcal{P}(n, m)|$ graphs with (i) no components isomorphic to $H$ and (ii) at least $\alpha n$ pendant edges.

We proceed as in the proof of Theorem 9 , and the counting is the same except that we now have $\omega(n)$ choices (instead of just $\Omega(n)$ ) for where to add the 'extra' edge after we have constructed the component isomorphic to $H$. Hence, we find that we can build $\left|\mathcal{G}_{n}\right| \omega(1)=|\mathcal{P}(n, m)| \omega(1)$ distinct graphs in $\mathcal{P}(n, m)$, which is a contradiction.

By using more precise estimates of add $(n, m)$, lower bounds for the number of components in $P_{n, m}$ isomorphic to $H$ can be obtained (see Theorem 38 of [1]). One such result is that $P_{n, m}$ will a.a.s. have linearly many components isomorphic to any given tree if we strengthen the upper bound on $\frac{m}{n}$ to $\lim \sup \frac{m}{n}<1$, rather than $\lim \sup \frac{m}{n} \leqslant 1$. Since this particular result shall be needed in Section 5, we will now provide a full proof. The method is exactly the same as with the last two results, but the equations involved are more complicated:

Theorem 11 Let $H$ be a (fixed) tree, let $c>0$ and $A<1$ be constants, and let $m(n) \in$ $[c n, A n]$ for all $n$. Then there exists a constant $\lambda(H, c, A)>0$ such that

$$
\begin{array}{r}
\mathbf{P}\left[P_{n, m} \text { will have less than } \lambda n \text { components isomorphic to } H\right]<e^{-\lambda n} \\
\text { for all large } n .
\end{array}
$$

Proof By Lemma 4, we know there exist constants $\alpha>0, \beta>0$ and $n_{0}$ such that $\mathbf{P}\left[P_{n, m}\right.$ will have less than $\alpha n$ pendant edges $]<e^{-\beta n}$ for all $n \geqslant n_{0}$. Let $\lambda$ be a small positive constant and suppose that there exists a value $n \geqslant n_{0}$ such that $\mathbf{P}\left[P_{n, m}\right.$ will have less than $\lceil\lambda n\rceil$ components isomorphic to $H] \geqslant e^{-\lceil\lambda n\rceil}$. Then there is a set $\mathcal{G}_{n}$ of at least a proportion $e^{-\lambda n}-e^{-\beta n}$ of the graphs in $\mathcal{P}(n, m)$ with (i) less than $\lambda n$ components isomorphic to $H$ and (ii) at least $\alpha n$ pendant edges.

Without loss of generality, we may assume that $\lambda$ is small enough and $n$ large enough that various inequalities hold during this proof. In particular, it is worth noting now that we may assume that $\alpha n \geqslant\lceil\lambda n\rceil|H|$ and $e^{-\lambda n}-e^{-\beta n} \geqslant \frac{1}{2} e^{-\lambda n}$.

To build graphs with at least $\lambda n$ components isomorphic to $H$, one can start with a graph $G \in \mathcal{G}_{n}\left(\left|\mathcal{G}_{n}\right|\right.$ choices), delete $\lceil\lambda n\rceil|H|$ pendant edges (at least $\left(\begin{array}{c}\lceil\alpha n\rceil \\ \lceil\lambda n\rceil|H|\end{array}\right)$ choices), and insert edges between $\lceil\lambda n\rceil|H|$ of the newly-isolated vertices (choosing one from each pendant edge) in such a way that they now form $\lceil\lambda n\rceil$ components isomorphic to $H$ (at least $\left(\begin{array}{c}\lceil\lambda n\rceil|H| \\ |H|, \ldots,|H|\end{array}\right) \frac{1}{\lceil\lambda n\rceil !}$ choices). We should then add $\lceil\lambda n\rceil$ edges somewhere in the rest of the graph (i.e. away from our newly constructed components) to maintain the correct number of edges overall (we have at least $\prod_{i=0}^{\lceil\lambda n\rceil-1} \operatorname{add}(n-\lceil\lambda n\rceil|H|, m-\lceil\lambda n\rceil|H|+i) \geqslant$ $(\operatorname{add}(n-\lceil\lambda n\rceil|H|, m))^{\lceil\lambda n\rceil}$ choices for this $)$. See Figure 4.

Hence, the number of ways that we have to build (not necessarily distinct) graphs in $\mathcal{P}(n, m)$ that have at least $\lambda n$ components isomorphic to $H$ is at least

$$
\frac{\lceil\alpha n\rceil !}{(\lceil\lambda n\rceil|H|) !(\lceil\alpha n\rceil-\lceil\lambda n\rceil|H|) !} \frac{(\lceil\lambda n\rceil|H|) !}{(|H| !)^{\lceil\lambda n\rceil}} \frac{1}{\lceil\lambda n\rceil !} \cdot(\operatorname{add}(n-\lceil\lambda n\rceil|H|, m))^{\lceil\lambda n\rceil}\left|\mathcal{G}_{n}\right|
$$




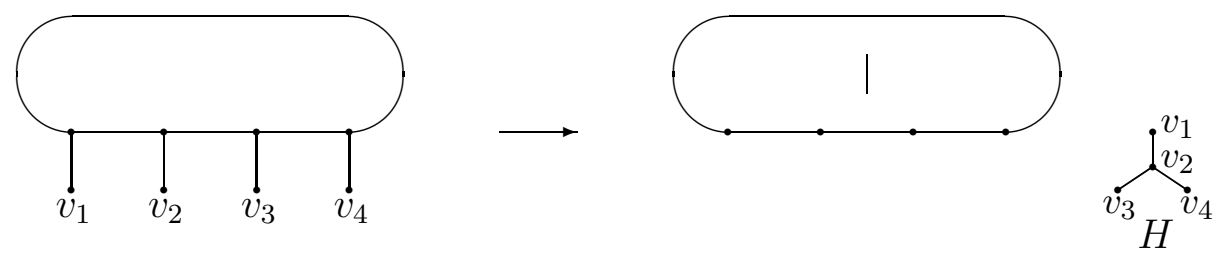

Figure 4: Constructing a component isomorphic to $H$.

$$
\begin{aligned}
& \geqslant(\lceil\alpha n\rceil-\lceil\lambda n\rceil|H|)^{\lceil\lambda n\rceil|H|}\left(\frac{1}{|H| !}\right)^{\lceil\lambda n\rceil} \frac{1}{\lceil\lambda n\rceil !} \cdot(\operatorname{add}(n-\lceil\lambda n\rceil|H|, m))^{\lceil\lambda n\rceil}\left|\mathcal{G}_{n}\right| \\
& \geqslant\left(\left(\frac{\alpha n}{2}\right)^{|H|}\left(\frac{1}{|H| !}\right)(\operatorname{add}(n-\lceil\lambda n\rceil|H|, m))\right)^{\lceil\lambda n\rceil} \frac{1}{\lceil\lambda n\rceil !}\left|\mathcal{G}_{n}\right|
\end{aligned}
$$

(since we may assume that $\lambda$ is sufficiently small and $n$ sufficiently large that $\left.\lceil\alpha n\rceil-\lceil\lambda n\rceil|H| \geqslant \frac{\alpha n}{2}\right)$.

Let us now consider the amount of double-counting:

Each of our constructed graphs will contain at most $\lceil\lambda n\rceil(|H|+3)-1$ components isomorphic to $H$ (since there were at most $\lceil\lambda n\rceil-1$ already in $G$; we have deliberately added $\lceil\lambda n\rceil$; and we may have created at most one extra one each time we deleted a pendant edge or added an edge in the rest of the graph), so we have at most $\left(\begin{array}{c}\lceil\lambda n\rceil(|H|+3)-1 \\ \lceil\lambda n\rceil\end{array}\right) \leqslant$ $\frac{1}{\lceil\lambda n\rceil !}(\lceil\lambda n\rceil(|H|+3))^{\lceil\lambda n\rceil}$ possibilities for which are our created components. We then have at most $n^{\lceil\lambda n\rceil|H|}$ possibilities for where the vertices in our created components were attached originally and at most $\left(\begin{array}{c}m \\ \lceil\lambda n\rceil\end{array}\right) \leqslant(3 n)^{\lceil\lambda n\rceil}$ possibilities for which edges was added. Thus, the amount of double-counting is at most $\frac{1}{\lceil\lambda n\rceil !}\left(\lceil\lambda n\rceil(|H|+3) n^{|H|} 3 n\right)^{\lceil\lambda n\rceil}$.

Hence, putting everything together, we find that the number of distinct graphs in $\mathcal{P}(n, m)$ that have at least $\lambda n$ components isomorphic to $H$ is at least

$$
\left(\left(\frac{\alpha}{2}\right)^{|H|} \frac{1}{|H| !}(\operatorname{add}(n-\lceil\lambda n\rceil|H|, m)) \frac{1}{\lceil\lambda n\rceil(|H|+3) 3 n}\right)^{\lceil\lambda n\rceil}\left|\mathcal{G}_{n}\right| .
$$

Recall that $m \leqslant A n$, where $A<1$. Thus, we may assume that $\lambda$ is sufficiently small and $n$ sufficiently large that $m \leqslant \frac{A+1}{2}(n-\lceil\lambda n\rceil|H|)$. Hence, by Lemma 7 , we have $\operatorname{add}(n-\lceil\lambda n\rceil|H|, m) \geqslant(1+o(1))\left(\frac{\left(1-\frac{A+1}{2}\right)^{2}}{2}\right) n^{2}=(1+o(1)) \frac{(1-A)^{2}}{8} n^{2}$.

Therefore, we find that the number of graphs in $\mathcal{P}(n, m)$ that have at least $\lambda n$ components isomorphic to $H$ is at least

$$
\left((1+o(1)) \frac{\alpha^{|H|}}{2^{|H|}|H| ! 3(|H|+3)}\left(\frac{(1-A)^{2}}{8 \lambda}\right)\right)^{\lceil\lambda n\rceil}\left|\mathcal{G}_{n}\right| .
$$

But this is more than $|\mathcal{P}(n, m)|$ for large $n$, if $\lambda$ is sufficiently small, since we recall that $\left|\mathcal{G}_{n}\right| \geqslant \frac{1}{2} e^{-\lambda n}|\mathcal{P}(n, m)|$. Thus, by proof by contradiction, it must be that $\mathbf{P}\left[P_{n, m}\right.$ will have less than $\lambda n$ components isomorphic to $H]<e^{-\lambda n}$ for all large $n$. 


\section{Components II: Upper Bounds}

In this section, we shall produce upper bounds for $\mathbf{P}:=\mathbf{P}\left[P_{n, m}\right.$ will have a component isomorphic to $H]$ to complement the lower bounds of Section 3 .

We will start with the case $0<\liminf \frac{m}{n} \leqslant \lim \sup \frac{m}{n} \leqslant 1$, for which we have seen $\mathbf{P} \rightarrow 1$ if $H$ is a tree and $\lim \inf \mathbf{P}>0$ if $H$ is unicyclic. In this section, we shall complete matters by showing $\mathbf{P} \rightarrow 0$ if $H$ is multicyclic (see Theorem 12) and $\lim \sup \mathbf{P}<1$ if $H$ is unicyclic (see Theorem 13).

We will then deal with the case when $\lim \inf \frac{m}{n}>1$, for which we have seen $\lim \inf \mathbf{P}>$ 0 for all connected planar $H$ if we also have $\lim \sup \frac{m}{n}<3$. By examining the probability that $P_{n, m}$ is connected, we will now show $\mathbf{P} \rightarrow 0$ if $\frac{m}{n} \rightarrow 3$ (see Theorem 14) and $\lim \sup \mathbf{P}<1$ if $\lim \inf \frac{m}{n}>1$ (see Theorem 16).

We start with our aforementioned result for multicyclic components when $\frac{m}{n} \leqslant 1+o(1)$ :

Theorem 12 Let $H$ be a (fixed) multicyclic connected planar graph and let $m(n) \leqslant$ $(1+o(1)) n$. Then

$$
\mathbf{P}\left[P_{n, m} \text { will have a component isomorphic to } H\right] \rightarrow 0 \text { as } n \rightarrow \infty \text {. }
$$

Proof Let $\mathcal{G}_{n}$ denote the set of graphs in $\mathcal{P}(n, m)$ with a component isomorphic to $H$. For each graph $G \in \mathcal{G}_{n}$, let us delete 2 edges from a component $H^{\prime}\left(=H_{G}^{\prime}\right)$ isomorphic to $H$ in such a way that we do not disconnect the component. Let us then insert one edge between a vertex in the remaining component and a vertex elsewhere in the graph. We have $|H|(n-|H|)$ ways to do this, and planarity is maintained. Let us then also insert one other edge into the graph, without violating planarity (see Figure 5). We have at least $(\operatorname{add}(n, m))=\omega(n)$ choices for where to place this second edge, by Lemma 6 . Thus, we can construct $\left|\mathcal{G}_{n}\right| \omega\left(n^{2}\right)$ (not necessarily distinct) graphs in $\mathcal{P}(n, m)$.

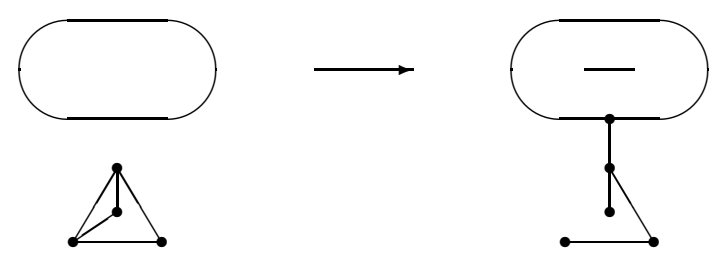

Figure 5: Redistributing edges from our multicyclic component.

Given one of our constructed graphs, there are $m=O(n)$ possibilities for the edge that was inserted last. There are then at most $m-1=O(n)$ possibilities for the other edge that was inserted. Since one of the two vertices incident with this edge must belong to $V\left(H^{\prime}\right)$, we then have at most two possibilities for $V\left(H^{\prime}\right)$ and then at most $\left(\begin{array}{c}\left(\begin{array}{c}|H| \\ 2 \\ 2\end{array}\right) \\ 2\end{array}\right)=O(1)$ possibilities for $E\left(H^{\prime}\right)$. Thus, we have built each graph at most $O\left(n^{2}\right)$ times, and so $\frac{\left|\mathcal{G}_{n}\right|}{|\mathcal{P}(n, m)|}=\frac{O\left(n^{2}\right)}{\omega\left(n^{2}\right)} \rightarrow 0$ 
We shall now look at unicyclic components. The basic argument will be the same as that of Theorem 12, i.e. we will start with $\mathcal{G}_{n}$, the set of graphs in $\mathcal{P}(n, m)$ with a component isomorphic to $H$, and redistribute edges from such components to construct other graphs in $\mathcal{P}(n, m)$. This time we will only be able to delete one edge from each component, and so we will only be able to show $\frac{\left|\mathcal{G}_{n}\right|}{|\mathcal{P}(n, m)|}=O(1)$, rather than $o(1)$. Hence, it will be crucial to keep track of the constants involved in the calculations, so that we can try to show $\lim \sup _{n \rightarrow \infty} \frac{\left|\mathcal{G}_{n}\right|}{|\mathcal{P}(n, m)|}<1$. Unfortunately, it turns out that the constants are actually only small enough for certain $H$, such as when $H$ is a cycle. However, it is fairly simple to relate the probability that a given component is isomorphic to $H$ to the probability that it is a cycle, and so we may deduce that the result actually holds for any unicyclic $H$.

Theorem 13 Let $H$ be a (fixed) unicyclic connected planar graph. Then, given any $m(n)$,

$$
\limsup _{n \rightarrow \infty} \mathbf{P}\left[P_{n, m} \text { will have a component isomorphic to } H\right]<1 \text {. }
$$

Proof Let $\mathcal{G}_{n}$ denote the set of graphs in $\mathcal{P}(n, m)$ with a component that is a cycle of order $|H|$. For each graph $G \in \mathcal{G}_{n}$, let us delete an edge from such a component, leaving a spanning path. Note that we have $|H|$ choices for this edge. Let us then insert an edge between a vertex in the spanning path and a vertex elsewhere (see Figure 6). We have $|H|(n-|H|)$ ways to do this and planarity is maintained. Thus, we have constructed $\left|\mathcal{G}_{n}\right||H|^{2}(n-|H|)$ graphs in $\mathcal{P}(n, m)$.

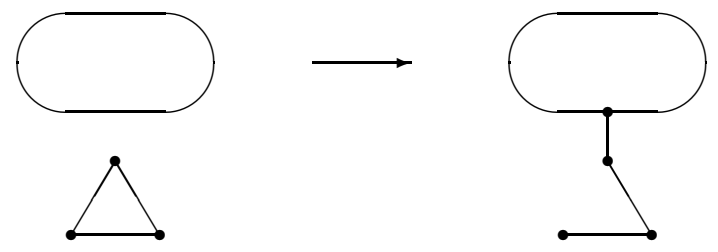

Figure 6: Redistributing an edge from our cycle.

Given one of our constructed graphs, there are at most $n-1$ possibilities for which is the inserted edge, since it must be a cut-edge. There are then at most 2 possibilities for the vertex set of our original cycle (at most one possibility for each endpoint of the edge), and then only one possibility for where the inserted edge was originally. Thus, we have built each graph at most $2(n-1)$ times. Therefore, $\left|\mathcal{G}_{n}\right| \leqslant \frac{2(n-1)}{|H|^{2}(n-|H|)}|\mathcal{P}(n, m)|$, and hence there exists an $\epsilon>0$ such that $\left|\mathcal{G}_{n}^{c}\right|>\epsilon|\mathcal{P}(n, m)|$ for all $n$, where $\mathcal{G}_{n}^{c}$ denotes $\mathcal{P}(n, m) \backslash \mathcal{G}_{n}$.

If $H$ is itself a cycle, then we are done. If not, let $r$ satisfy $\frac{|H| !}{r} \leqslant \frac{\epsilon}{2}$ and let $\mathcal{J}_{n}$ denote the set of graphs in $\mathcal{P}(n, m)$ with more than $r$ components isomorphic to $H$. Let us now consider the set $\mathcal{J}_{n} \cap \mathcal{G}_{n}^{c}$, i.e. the set of graphs in $\mathcal{P}(n, m)$ with more than $r$ components isomorphic to $H$, but no components that are cycles of order $|H|$.

For each graph $J \in \mathcal{J}_{n} \cap \mathcal{G}_{n}^{c}$, delete a component isomorphic to $H$ (we have more than $r$ choices for this) and replace it with a cycle of order $|H|$. Note that each graph is built at most $|H|$ ! times, since we will be able to see exactly where the modified component 
is, and so we can construct at least $\frac{r}{|H| !}\left|\mathcal{J}_{n} \cap \mathcal{G}_{n}^{c}\right|$ distinct graphs in $\mathcal{P}(n, m)$. Hence, $\left|\mathcal{J}_{n} \cap \mathcal{G}_{n}^{c}\right| \leqslant \frac{|H| !}{r}|\mathcal{P}(n, m)| \leqslant \frac{\epsilon}{2}|\mathcal{P}(n, m)|$.

Finally, let $\mathcal{J}_{n}^{c}$ denote $\mathcal{P}(n, m) \backslash \mathcal{J}_{n}$ and let us consider the set $\mathcal{J}_{n}^{c} \cap \mathcal{G}_{n}^{c}$ (i.e. the set of graphs in $\mathcal{P}(n, m)$ with at most $r$ components isomorphic to $H$ and no components that are cycles of order $|H|)$. Note that we have $\left|\mathcal{J}_{n}^{c} \cap \mathcal{G}_{n}^{c}\right|=\left|\mathcal{G}_{n}^{c}\right|-\left|\mathcal{J}_{n} \cap \mathcal{G}_{n}^{c}\right| \geqslant \frac{\epsilon}{2}|\mathcal{P}(n, m)|$, by the previous paragraph.

For each graph $L \in \mathcal{J}_{n}^{c} \cap \mathcal{G}_{n}^{c}$, let us change each component isomorphic to $H$ into a cycle. The amount of double-counting will be at most $(|H| !)^{r}$, since we will be able to see exactly where the modified components are, and so we can construct at least $\frac{\left|\mathcal{J}_{n}^{c} \cap \mathcal{G}_{n}^{c}\right|}{(|H| !)^{r}} \geqslant \frac{\epsilon|\mathcal{P}(n, m)|}{2(|H| !)^{r}}$ distinct graphs in $\mathcal{P}(n, m)$ without any components isomorphic to $H$.

As mentioned at the start of this section, we shall now obtain upper bounds for $\mathbf{P}\left[P_{n, m}\right.$ will have a component isomorphic to $\left.H\right]$ when $\lim \inf \frac{m}{n}>1$ by investigating the probability that $P_{n, m}$ will be connected, in which case it clearly won't contain any components of order less than $n$ at all.

We start with the case when $\frac{m}{n} \rightarrow 3$ :

Theorem 14 Let $m(n)=3 n-o(n)$. Then

$$
\mathbf{P}\left[P_{n, m} \text { will be connected }\right] \rightarrow 1 \text { as } n \rightarrow \infty .
$$

Proof Let $G \in \mathcal{P}(n, m)$ and let us consider how many triangles in $G$ contain at least one vertex with degree $\leqslant 6$. We shall call such triangles 'good' triangles.

First, note that (assuming $n \geqslant 3) G$ may be extended to a triangulation by inserting $3 n-6-m=o(n)$ 'phantom' edges. Let $d_{i}$ denote the number of vertices of degree $i$ in such a triangulation. Then $7 \sum_{i \geqslant 7} d_{i} \leqslant \sum_{i \geqslant 1} i d_{i}=2(3 n-6)$. Thus, $\sum_{i \geqslant 7} d_{i}<\frac{6 n}{7}$ and so $\sum_{i \leqslant 6} d_{i}>\frac{n}{7}$.

For $n>3$, each of these vertices of small degree will be in at least three faces of the triangulation, all of which will be good triangles. This counts each good triangle at most three times (once for each vertex), so our triangulation must have at least $3 \cdot \frac{n}{7} \cdot \frac{1}{3}=\frac{n}{7}$ good triangles that are faces. Each of our phantom edges is in exactly two faces of the triangulation, so our original graph $G$ must contain at least $\frac{n}{7}+o(n)$ of our good triangles (note that these triangles will still be 'good', since the degrees of the vertices will be at most what they were in the triangulation).

We will now consider how many cut-edges a graph in $\mathcal{P}(n, m)$ may have. If we delete all $c$ cut-edges, then the remaining graph will consist of $b$, say, blocks, each of which is either 2-edge-connected or is an isolated vertex. Note that the graph formed by condensing each block to a single node and re-inserting the cut-edges must be acyclic, so $c \leqslant b-1$. Label the blocks $1,2, \ldots b$ and let $n_{i}$ denote the number of vertices in block $i$. Then the number of edges in block $i$ is at most $3 n_{i}-6$ if $n_{i} \geqslant 3$ and is $0=3 n_{i}-3$ otherwise (since $n_{i}<3$ implies that $\left.n_{i}=1\right)$. Thus, $m \leqslant \sum_{i=1}^{b}\left(3 n_{i}-3\right)+c=3 n-3 b+c<3 n-2 c$, and so $c<\frac{3 n-m}{2}=o(n)$.

We now come to the main part of the proof. Let $\mathcal{G}_{n}$ denote the set of graphs in $\mathcal{P}(n, m)$ that are not connected, and choose a graph $G \in \mathcal{G}_{n}$. Choose a good triangle in $G$ (at 
least $\frac{n}{7}+o(n)$ choices) and delete an edge that is opposite a vertex with degree $\leqslant 6$. Then insert an edge between two vertices in different components (we have $a$, say, choices for this edge). See Figure 7.
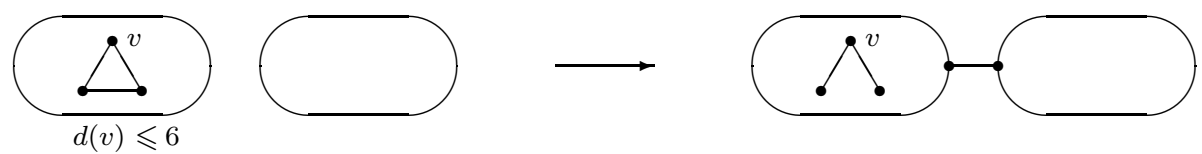

Figure 7: Constructing our new graph.

As mentioned in the previous proof, the number of possible edges between disjoint sets $X$ and $Y$ is $|X||Y|$ and if $|X| \leqslant|Y|$ then $|X||Y|>(|X|-1)(|Y|+1)$, so it follows that the number of choices for the edge to insert is minimized when we have one isolated vertex and one component of $n-1$ vertices. Thus, $a \geqslant n-1$ and so we have created at least $\left|\mathcal{G}_{n}\right|\left(\frac{n}{7}-o(n)\right)(n-1)$ graphs in $\mathcal{P}(n, m)$.

Given one of our created graphs, there are at most $o(n)$ possibilities for which edge was inserted, since it must be a cut-edge. There are then at most $\left(\begin{array}{l}6 \\ 2\end{array}\right) n$ possibilities for where the deleted edge was originally, since it must have been between two neighbours of a vertex with degree $\leqslant 6$ (we have at most $n$ possibilities for this vertex and then at most $\left(\begin{array}{l}6 \\ 2\end{array}\right)$ possibilities for its neighbours). Hence, we have built each graph at most $o\left(n^{2}\right)$ times.

Thus, $|\mathcal{P}(n, m)| \geqslant \frac{\left(\frac{1}{7}\right) n^{2}+o\left(n^{2}\right)}{o\left(n^{2}\right)}\left|\mathcal{G}_{n}\right|$, and so $\frac{\left|\mathcal{G}_{n}\right|}{|\mathcal{P}(n, m)|} \leqslant \frac{o\left(n^{2}\right)}{\Theta\left(n^{2}\right)} \rightarrow 0$.

It now only remains to look at when $1<\lim \inf \frac{m}{n} \leqslant \lim \sup \frac{m}{n}<3$.

In [5], generating function techniques were used to produce rather precise asymptotic estimates for both $|\mathcal{P}(n,\lfloor q n\rfloor)|$ and $\left|\mathcal{P}_{c}(n,\lfloor q n\rfloor)\right|$, the number of connected graphs in $\mathcal{P}(n,\lfloor q n\rfloor)$, when $q \in(1,3)$ :

Proposition 15 ([5], Theorem 3) Let $q \in(1,3)$ be a constant. Then

$$
\begin{aligned}
|\mathcal{P}(n,\lfloor q n\rfloor)| & \sim g(q) \cdot(u(q))^{q n-\lfloor q n\rfloor} \cdot n^{-4} \gamma(q)^{n} n ! \\
\text { and }\left|\mathcal{P}_{c}(n,\lfloor q n\rfloor)\right| & \sim g_{c}(q) \cdot(u(q))^{q n-\lfloor q n\rfloor} \cdot n^{-4} \gamma(q)^{n} n !,
\end{aligned}
$$

where $g(q), g_{c}(q), u(q)$ and $\gamma(q)$ are computable analytic functions.

Clearly, Proposition 15 may be used to obtain the exact asymptotic limit for $\mathbf{P}\left[P_{n,\lfloor q n}\right\rfloor$ will be connected] when $q \in(1,3)$, and it turns out that this limit is strictly greater than 0 for all $q>1$. It is no surprise that this result holds uniformly, and a combinatorial proof is given in [1]. Hence, we have

Theorem 16 ([1], Theorem 44) Let $b>1$ be a constant and let $m(n) \in[b n, 3 n-6]$. Then there exists a constant $c(b)>0$ such that

$$
\mathbf{P}\left[P_{n, m} \text { will be connected }\right]>c \text { for all } n \text {. }
$$


Note that we now have a complete description of $\mathbf{P}\left[P_{n, m}\right.$ will have a component isomorphic to $H$ ], in terms of exactly when it is/isn't bounded away from 0 and/or 1 . By combining Theorems 8 and 10 with Theorem 16, we also have a complete picture of $\mathbf{P}\left[P_{n, m}\right.$ will be connected].

\section{Subgraphs}

We have now finished looking at the probability that $P_{n, m}$ will have a component isomorphic to $H$. In this section, we will instead investigate $\mathbf{P}^{\prime}:=\mathbf{P}\left[P_{n, m}\right.$ will have a subgraph isomorphic to $H$ ]. We already know from Lemma 3 that $\mathbf{P}^{\prime} \rightarrow 1$ for all $H$ if $1<\liminf \frac{m}{n} \leqslant \limsup \frac{m}{n}<3$, and we shall soon see (in Theorem 17) that we can actually drop the $\lim \sup \frac{m}{n}<3$ condition. Hence, the interest lies with the case when $0<\liminf \frac{m}{n} \leqslant \lim \sup \frac{m}{n} \leqslant 1$.

It follows from our results on components that $\mathbf{P}^{\prime} \rightarrow 1$ for this region if $H$ is a tree and that $\lim \sup \mathbf{P}^{\prime}>0$ if $H$ is a connected unicyclic graph. In this section, we shall see for the latter case that $\lim \sup \mathbf{P}^{\prime}<1$ if $\lim \sup \frac{m}{n}<1$ (see Theorem 18), but that $\mathbf{P}^{\prime} \rightarrow 1$ if $\frac{m}{n} \rightarrow 1$ (see Theorem 21). If $H$ is a connected multicyclic graph, we shall see (in Theorem 22) that $\mathbf{P}^{\prime} \rightarrow 0$ if $\lim \sup \frac{m}{n}<1$. This leaves the remaining unanswered question of what happens when $H$ is multicyclic and $\frac{m}{n} \rightarrow 1$.

We now start with a short discussion of the case when $\frac{m}{n} \rightarrow 3$. Since we know from Lemma 3 that $\mathbf{P}\left[P_{n, m}\right.$ will have a copy of $\left.H\right] \rightarrow 1$ as $n \rightarrow \infty$ for all planar $H$ if $1<\liminf \frac{m}{n} \leqslant \limsup \frac{m}{n}<3$, it is intuitive that the result ought to also hold when $\frac{m}{n} \rightarrow 3$. Clearly, we wouldn't expect to have any appearances of $H$ if $m$ is close to $3 n-6$, since appearances involve cut-edges, but it turns out that the same proof does work if we replace appearances of $H$ by '6-appearances' of triangulations containing $H$, where a 6 appearance is similar to an appearance but with six edges (from three vertices) connecting it to the rest of the graph instead of just one (see [1] for details).

Hence, we obtain:

Theorem 17 ([1], Theorem 61) Let $H$ be a (fixed) planar graph and let $m(n)$ satisfy $\liminf _{n \rightarrow \infty} \frac{m}{n}>1$. Then there exists a constant $\alpha>0$ such that

$$
\mathbf{P}\left[P_{n, m} \text { will not have a set of at least } \alpha \text { copies of } H\right]=e^{-\Omega(n)} \text {. }
$$

We will now spend the remainder of this paper looking at the case when we have $\frac{m}{n} \leqslant 1+o(1)$. We start with an upper bound for unicyclic graphs when $\lim \sup \frac{m}{n}<1$ :

Theorem 18 Let $H$ be a (fixed) unicyclic connected planar graph, let $A<1$ be a constant, and let $m(n) \leqslant$ An for all $n$. Then

$$
\limsup _{n \rightarrow \infty} \mathbf{P}\left[P_{n, m} \text { will have a copy of } H\right]<1 \text {. }
$$


Sketch of Proof Let $C$ denote the unique cycle in $H$. Clearly, it suffices to show $\limsup _{n \rightarrow \infty} \mathbf{P}\left[P_{n, m}\right.$ will have a copy of $\left.C\right]<1$.

By Theorems 11 and 13, we know that there must be a decent proportion of graphs with lots of isolated vertices, but no components isomorphic to $C$. If such a graph contains lots of copies of $C$, then we may 'transfer' the edges of one of these to some isolated vertices to construct a component isomorphic to $C$, and the amount of double-counting will be small since there were no such components originally. Hence, we can use this idea to show that the proportion of graphs with lots of copies of $C$, lots of isolated vertices and no components isomorphic to $C$ must be small, and so there must be a decent proportion of graphs with few copies of $C$ (and lots of isolated vertices and no components isomorphic to $C)$.

Given such a graph, we may then destroy the few copies of $C$ by deleting the edges from them and inserting edges between components of the graph.

Full Proof As mentioned in the sketch-proof, it clearly suffices for us to show that $\limsup _{n \rightarrow \infty} \mathbf{P}\left[P_{n, m}\right.$ will have a copy of $\left.C\right]<1$, where $C$ denotes the unique cycle in $H$.

Let $b \in\left(0, \frac{1}{2}\right)$ be a fixed constant and suppose $m \in[b n, A n]$ for all $n$. Then, by Theorem 11 (with $H$ as an isolated vertex), there exists a constant $\beta>0$ such that $\mathbf{P}\left[P_{n, m}\right.$ will have at least $\beta n$ isolated vertices $] \rightarrow 1$ as $n \rightarrow \infty$. Clearly, $P_{n, m}$ must also have at least $n-2 b n$ isolated vertices if $m \leqslant b n$, so by setting $\alpha=\min \{\beta, 1-2 b\}$ we find that $\mathbf{P}\left[P_{n, m}\right.$ will have at least $\alpha n$ isolated vertices $] \rightarrow 1$ as $n \rightarrow \infty$ whenever $m \leqslant A n$.

Let $\mathcal{G}_{n}$ denote the set of graphs in $\mathcal{P}(n, m)$ with at least $\alpha n$ isolated vertices and no components isomorphic to $C$. Then, using Theorem 13, we now know that there exists an $\epsilon>0$ such that $\left|\mathcal{G}_{n}\right| \geqslant \epsilon|\mathcal{P}(n, m)|$ for all sufficiently large $n$.

Let $r$ satisfy $\frac{(|C|+1) !}{r \alpha|C|}<\frac{\epsilon}{2}$ and let $\mathcal{J}_{n}$ denote the set of graphs in $\mathcal{P}(n, m)$ whose maximal number of edge-disjoint copies of $C$ is more than $r$. Let us now consider the set $\mathcal{J}_{n} \cap \mathcal{G}_{n}$, i.e. the set of graphs in $\mathcal{P}(n, m)$ with at least $\alpha n$ isolated vertices, more than $r$ edge-disjoint copies of $C$, but no components isomorphic to $C$.

For each graph $J \in \mathcal{J}_{n} \cap \mathcal{G}_{n}$, delete all $|C|$ edges from a copy of $C$ (we have more than $r$ choices for this) and insert them between $|C|$ isolated vertices (we have at least $\left(\begin{array}{l}\alpha n \\ |C|\end{array}\right)$ choices for these) to form a component isomorphic to $C$ (see Figure 8). We can thus construct at least $\left|\mathcal{J}_{n} \cap \mathcal{G}_{n}\right| r\left(\begin{array}{c}\alpha n \\ |C|\end{array}\right)$ graphs in $\mathcal{P}(n, m)$.

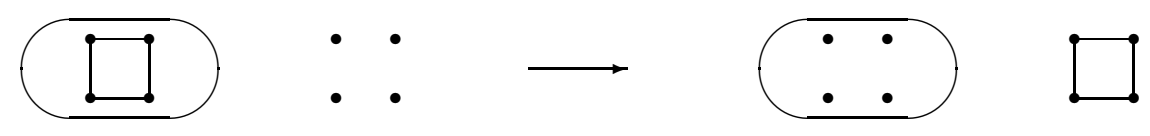

Figure 8: Constructing a component isomorphic to $C$.

Given one of our constructed graphs, there are at most $|C|+1$ components isomorphic to $C$, since we have deliberately constructed one and we may have also created at most $|C|$ when we deleted the edges (since each vertex in the deleted copy of $C$ might now be in a component isomorphic to $C$ ). Thus, there are at most $|C|+1$ possibilities for which is 
the deliberately constructed component and hence at most $|C|+1$ possibilities for which are the inserted edges. There are then at most $\left(\begin{array}{c}n \\ |C|\end{array}\right)|C| ! \leqslant n^{|C|}$ possibilities for where these edges were originally. Thus, we have built each graph at most $(|C|+1) n^{|C|}$ times and so

$$
\begin{aligned}
\left|\mathcal{J}_{n} \cap \mathcal{G}_{n}\right| & <\frac{(|C|+1) n^{|C|}|\mathcal{P}(n, m)|}{r\left(\begin{array}{l}
\alpha n \\
|C|
\end{array}\right)} \\
& =(1+o(1)) \frac{(|C|+1) !}{r \alpha^{|C|}}|\mathcal{P}(n, m)| \\
& <\frac{\epsilon}{2}|\mathcal{P}(n, m)| \text { for sufficiently large } n \text {, by definition of } r .
\end{aligned}
$$

Thus, $\left|\mathcal{J}_{n}^{c}\right| \geqslant\left|\mathcal{J}_{n}^{c} \cap \mathcal{G}_{n}\right|=\left|\mathcal{G}_{n}\right|-\left|\mathcal{J}_{n} \cap \mathcal{G}_{n}\right|>\frac{\epsilon}{2}|\mathcal{P}(n, m)|$, where $\mathcal{J}_{n}^{c}$ denotes $\mathcal{P}(n, m) \backslash \mathcal{J}_{n}$ (i.e. the collection of graphs in $\mathcal{P}(n, m)$ without a set of more than $r$ edge-disjoint copies of $C$ ).

For $L \in \mathcal{J}_{n}^{c}$, let $S(L)$ denote a maximal set of edge-disjoint copies of $C$ (so $|S(L)| \leqslant r$ ) and, for $s \leqslant r$, let $\mathcal{J}_{n, s}$ denote the set of graphs in $\mathcal{P}(n, m)$ with $|S(L)|=s$. Let us now consider the set $\mathcal{J}_{n, s}$.

For each graph $L \in \mathcal{J}_{n, s}$, delete all $|C|$ edges from a copy of $C$ that is in $S(L)$. Note that the graphs will now have $|S|=s-1$, by maximality of $S(L)$. Clearly, we may insert an edge between any two vertices in different components without introducing a copy of a cycle, and, as in the proof of Lemma 7 , we have at least $(1+o(1)) \frac{(1-A)^{2}}{2} n^{2}$ choices for how to do this. Thus, inserting $|C|$ edges, we find that we may construct at least $(1+o(1)) \frac{\left(\frac{(1-A)^{2}}{2}\right)^{|C|} n^{2|C|}}{|C| !}\left|\mathcal{J}_{n, s}\right|$ graphs in $\mathcal{J}_{n, s-1}$ (see Figure 9$)$.

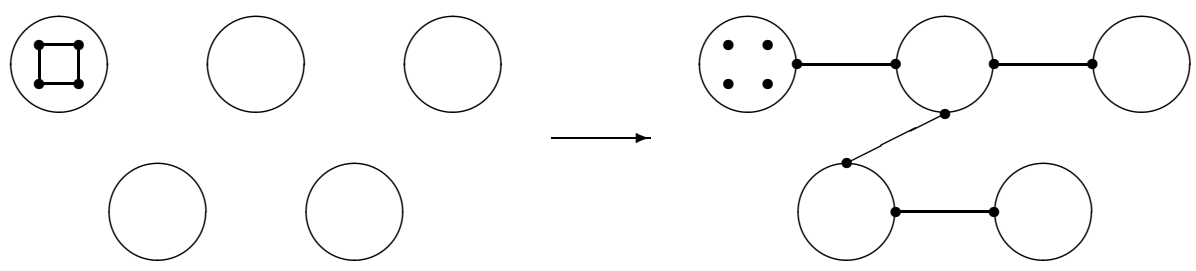

Figure 9: Destroying a copy of $C$.

Given one of our created graphs, there are at most $\left(\begin{array}{c}m \\ |C|\end{array}\right) \leqslant(A n)^{|C|}$ possibilities for which edges were inserted and at most $\left(\begin{array}{c}n \\ |C|\end{array}\right)|C| !<n^{|C|}$ possibilities for where they were originally. Thus, we have built each graph at most $A^{|C|} n^{2|C|}$ times, and so $\left|\mathcal{J}_{n, s-1}\right| \geqslant$ $(1+o(1)) \frac{\left(\frac{(1-A)^{2}}{2 A}\right)^{|C|}}{|C| !}\left|\mathcal{J}_{n, s}\right|$.

For $s \leqslant r$, let $\mathcal{J}_{n, \leqslant s}$ denote the set of graphs in $\mathcal{P}(n, m)$ with $|S| \leqslant s$. Then, with 
$z=\frac{\left(\frac{(1-A)^{2}}{2 A}\right)^{|C|}}{|C| !}$, we have $\left|\mathcal{J}_{n, \leqslant s-1}\right| \geqslant(1+o(1)) \frac{z}{1+z}\left|\mathcal{J}_{n, \leqslant s}\right|$ for all $s \leqslant r$. Thus, we obtain

$$
\begin{aligned}
\left|\mathcal{J}_{n, \leqslant 0}\right| & \geqslant(1+o(1))^{r}\left(\frac{z}{1+z}\right)^{r}\left|\mathcal{J}_{n, \leqslant r}\right| \\
& =(1+o(1))\left(\frac{z}{1+z}\right)^{r}\left|\mathcal{J}_{n}^{c}\right| \\
& \geqslant(1+o(1))\left(\frac{z}{1+z}\right)^{r} \frac{\epsilon}{2}|\mathcal{P}(n, m)|
\end{aligned}
$$

But $\mathcal{J}_{n, \leqslant 0}$ is the set of graphs in $\mathcal{P}(n, m)$ without a copy of $C$, and so we are done.

In a moment, we shall complete the unicyclic case by looking at what happens when $\frac{m}{n} \rightarrow 1$. First, though, we need to note two useful lemmas on $\kappa\left(P_{n, m}\right)$, the number of components of $P_{n, m}$ :

Lemma 19 ([1], Lemma 41) Let $m(n) \in[n, 3 n-6]$ for all large $n$. Then there exists a constant $c$ such that

$$
\mathbf{P}\left[\kappa\left(P_{n, m}\right)>\left\lceil\frac{c n}{\ln n}\right\rceil\right]=e^{-\Omega(n)} .
$$

Proof The proof follows that of Lemma 2.6 of [4], which deals with the case when $m=\lfloor q n\rfloor$ for fixed $q \in[1,3)$ (see [1] for details).

Lemma 20 ([1], Proposition 50) Let $q \in(0,1)$ be a constant and let $m(n) \in[q n, n-1]$ for all large $n$. Then there exists a constant $c=c(q)$ such that

$$
\mathbf{P}\left[\kappa\left(P_{n, m}\right)>\left\lceil\frac{c n}{\ln n}+n-m\right\rceil\right]=e^{-\Omega(n)} .
$$

Proof The proof follows that of Lemma 6.6 of [3], which deals with the case when $m=n-(\beta+o(1))(n / \ln n)$ for fixed $\beta>0$ (see [1] for details).

We may now prove our aforementioned result for when $\frac{m}{n} \rightarrow 1$ :

Theorem 21 Let $H$ be a (fixed) connected unicyclic graph and let $m(n)=(1+o(1)) n$. Then

$$
\mathbf{P}\left[P_{n, m} \text { will have a copy of } H\right] \rightarrow 1 \text { as } n \rightarrow \infty \text {. }
$$

Sketch of Proof We work with the set of graphs that don't have any copies of $H$. By Lemma 4, we may assume that we have lots of pendant edges, so we may delete $|H|$ of these edges and then use them with $|H|-1$ of the associated (now isolated) vertices to convert another pendant edge into an appearance of $H$ (see Figure 10). Note that we are also left with an extra isolated vertex. 
By Lemmas 19 and 20, we may assume that there are not very many isolated vertices. Thus, since our original graphs had no appearances of $H$, the amount of double-counting will be small, and hence the size of our original set of graphs must have been small.

Full Proof Let $\mathcal{G}_{n}$ denote the set of graphs in $\mathcal{P}(n, m)$ with no copies of $H$, and let $X$ denote the event that $P_{n, m} \in \mathcal{G}_{n}$.

Recall, from Lemma 4, that there exists an $\alpha>0$ such that

$$
\mathbf{P}\left[P_{n, m} \text { will have at least } \alpha n \text { pendant edges }\right] \rightarrow 1 \text { as } n \rightarrow \infty .
$$

Let $\mathcal{H}_{n}$ denote the set of graphs in $\mathcal{P}(n, m)$ with at least $\alpha$ n pendant edges, and let $Y$ denote the event that $P_{n, m} \in \mathcal{H}_{n}$.

Also, note that by Lemmas 19 and 20, there exists a $c>0$ such that

$$
\mathbf{P}\left[\kappa\left(P_{n, m}\right)>2 \max \left\{\frac{c n}{\ln n}, n-m\right\}\right]=e^{-\Omega(n)} .
$$

Let $x=x(n)=2 \max \left\{\frac{c n}{\ln n}, n-m\right\}=o(n)$, let $\mathcal{I}_{n}$ denote the number of graphs in $\mathcal{P}(n, m)$ with at most $x$ components, and let $Z$ denote the event that $P_{n, m} \in \mathcal{I}_{n}$.

Note that $\mathbf{P}[X] \leqslant \mathbf{P}[X \cap Y \cap Z]+\mathbf{P}[\bar{Y}]+\mathbf{P}[\bar{Z}] \rightarrow \mathbf{P}[X \cap Y \cap Z]$ as $n \rightarrow \infty$, by (1) and (2). Thus, it suffices to show that $\mathbf{P}[X \cap Y \cap Z] \rightarrow 0$ as $n \rightarrow \infty$.

Given a graph in $\mathcal{G}_{n} \cap \mathcal{H}_{n} \cap \mathcal{I}_{n}$, let us choose $|H|+1$ pendant edges (we have at least $\left(\begin{array}{c}a n \\ |H|+1\end{array}\right)$ choices for these). We shall use these edges to create an appearance of $H$.

Out of our chosen $|H|+1$ pendant edges, let the edge incident with the lowest labelled vertex of degree 1 be called 'special', and let this associated lowest labelled vertex of degree 1 be called the 'root'. Let us delete our $|H|$ non-special pendant edges to create at least $|H|$ isolated vertices.

We may choose $|H|-1$ of these newly isolated vertices in such a way that no two were adjacent in the original graph (i.e. we don't choose two vertices from the same pendant edge, even if that is possible). We may then use these chosen isolated vertices, together with the root, to construct an appearance of $H$ by inserting $|H|$ edges appropriately (see Figure 10).
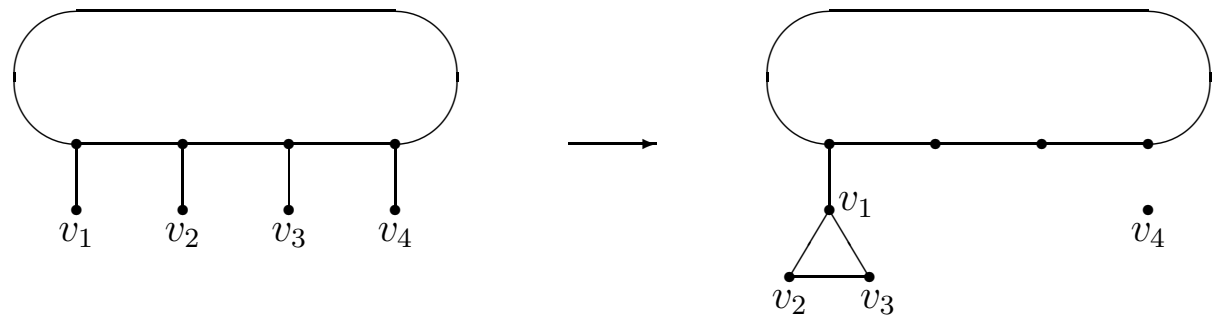

Figure 10: Constructing an appearance of $H$.

We shall now consider the amount of double-counting:

Recall that our original graphs contained no copies of $H$ and note that we cannot have created any copies by deleting edges. As observed in the proof of Theorem 4.1 of [7], an 
appearance of $H$ meets at most $|H|-1$ other appearances of $H$ (since there are at most $|H|-1$ cut-edges in $H$ and each of these can have at most one 'orientation' that provides an appearance of $H$ ). Thus, when we deliberately constructed our appearance of $H$ we can have only created at most $|H|$ appearances of $H$ in total.

Therefore, given one of our constructed graphs, there are at most $|H|$ possibilities for which is our deliberately created appearance of $H$. We may then recover the original graph by deleting the $|H|$ edges from this appearance, joining the $|H|-1$ non-root vertices back to the rest of the graph (at most $n^{|H|-1}$ possibilities), and joining the correct isolated vertex back to the rest of the graph (at most in possibilities, where $i$ denotes the number of isolated vertices in the constructed graph).

We know that the number of components in the original graph was at most $x$, and each time we deleted an edge we can have only increased the number of components by at most 1 . Thus, the number of components in our constructed graph is at most $x+|H|$, and so $i \leqslant x+|H|$.

Thus, we have built each graph at most $|H| n^{|H|-1}(x+|H|) n$ times.

Therefore,

$$
\begin{aligned}
\mathbf{P}[X \cap Y \cap Z] & =\frac{\left|\mathcal{G}_{n} \cap \mathcal{H}_{n} \cap \mathcal{I}_{n}\right|}{|\mathcal{P}(n, m)|} \\
& \leqslant \frac{|H| n^{|H|}(x+|H|)}{\left(\begin{array}{c}
\alpha n \\
|H|+1
\end{array}\right)} \\
& =\frac{o\left(n^{|H|+1}\right)}{\Theta\left(n^{|H|+1}\right)}, \text { since } x=o(n) \\
& \rightarrow 0 \text { as } n \rightarrow \infty .
\end{aligned}
$$

It now only remains to look at the case when $\frac{m}{n} \leqslant 1+o(1)$ and $H$ is a connected multicyclic graph. If $\lim \sup \frac{m}{n}<1$, we may prove the following:

Theorem 22 Let $H$ be a (fixed) multicyclic connected planar graph and let $m(n)$ satisfy $\limsup _{n \rightarrow \infty} \frac{m}{n}<1$. Then

$$
\mathbf{P}\left[P_{n, m} \text { will have a copy of } H\right] \rightarrow 0 \text { as } n \rightarrow \infty \text {. }
$$

Proof Let $\mathcal{G}_{n}$ denote the set of graphs in $\mathcal{P}(n, m)$ with a copy of $H$. For each graph $G \in \mathcal{G}_{n}$, let us delete all $e(H)$ edges from a copy of $H$ and then insert these edges back into the graph (see Figure 11). By Lemma 7, we have $\Theta\left(n^{2 e(H)}\right)$ ways to do this,

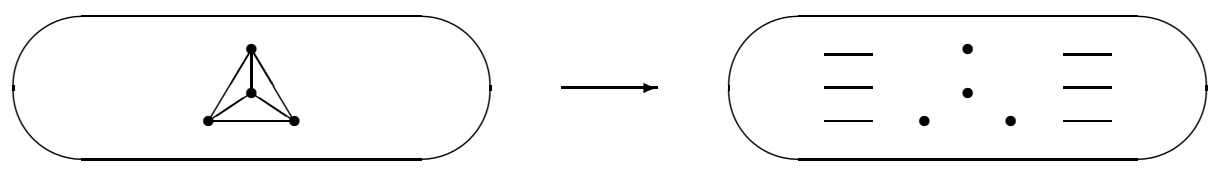

Figure 11: Redistributing the edges of $H$.

maintaining planarity. 
There are then $\left(\left(\begin{array}{c}n \\ |H|\end{array}\right) \frac{|H| !}{|\operatorname{Aut}(H)|}\right)=O\left(n^{|H|}\right)$ possibilities for where the copy of $H$ was originally and $\left(\begin{array}{c}m \\ e(H)\end{array}\right)=O\left(n^{e(H)}\right)$ possibilities for which edges were inserted, so we have built each graph $O\left(n^{(|H|+e(H))}\right)$ times.

Therefore, $\frac{\left|\mathcal{G}_{n}\right|}{|\mathcal{P}(n, m)|}=O\left(\frac{n^{(|H|+e(H))}}{n^{2 e(H)}}\right) \rightarrow 0$, since $e(H)>|H|$.

By using more precise estimates of $\operatorname{add}(n, m)$, it is possible to slightly increase the upper bound imposed on $m$ in Theorem 22 to include some functions $m(n)$ for which $\frac{m}{n}$ converges to 1 slowly (see Theorems 68 and 70 of [1]). However, we do not yet have a result that covers the whole region $m=(1+o(1)) n$.

\section{Acknowledgements}

I am very grateful to Colin McDiarmid for his advice and helpful suggestions.

\section{References}

[1] C. Dowden, Uniform random planar graphs with degree constraints, DPhil thesis (2008), available at http://ora.ouls.ox.ac.uk.

[2] S. Gerke, C. McDiarmid, On the number of edges in random planar graphs, Combinatorics, Probability and Computing 13 (2004), 165-183.

[3] S. Gerke, C. McDiarmid, A. Steger, A. Weißl, Random planar graphs with given average degree, from Combinatorics, Complexity and Chance, Oxford University Press (2007), 83-102.

[4] S. Gerke, C. McDiarmid, A. Steger, A. Weißl, Random planar graphs with n nodes and a fixed number of edges, Proceedings of the ACM-SIAM Symposium on Discrete Algorithms (2005), 999-1007.

[5] O. Giménez, M. Noy, Asymptotic enumeration and limit laws of planar graphs, Journal of the American Mathematical Society 22 (2009), 309-329.

[6] S. Janson, T. Łuczak, A. Ruciński, Random Graphs, Wiley (2000).

[7] C. McDiarmid, A. Steger, D. Welsh, Random planar graphs, Journal of Combinatorial Theory Series B 93 (2005), 187-205. 